\section{Microscope Instrumentation and Optics: A Researcher's "Road Test"}

\section{Dr. Robert F. Smith, DIP.RMS, HFRAS, FRMS}

Microscopists in today's laboratories face multiple challenges. Many must examine specimens according to legal, governmental or grant-specific requirements. Protocol requires that the specimens be documented by conventional photography, electronic imaging techniques, or both. Because of the thousands of specimens examined, operators work under conditions of potential physical strain for hours at a time. And the material they examine requires that they have expertise with large and complex imaging instruments.

Truthfully, up to now, their instruments have not always been easy partners to work with. Most research microscopes in the field today have extensive limitations of one kind or another. Taking all this into account, lab directors and microscopists in a recent poll revealed the following "wish listt" of prerequisites for research microscopes:

- Offers magnifications ranging from macro $(1 \mathrm{X})$ all the way through to high magnification without having to significantly change the instru ment set-up.

- Is capable of handling all illumination techniques, l.e. brightfield, darkfield, phase contrast, differential interference contrast (DIC) and epi-fluorescence; without the time-consuming drudgery of changing a lot of accessories and optics, and realigning the optical system.

- Has a well-thought-out ergonomic design that does not impose physical stress on the operator, and enhances the operation of the instrument.

\section{- Offers extraordinary optical quality and documentation capability.}

But until recently, no such instrument existed. Lately, Nikon's Eclipse CFl60 series of microscopes were evaluated against this users' list of prerequisites. The "road test" that the research-grade E800 instruments were put through may prove enlightening for scientists whose research requirements are similarly complex

\section{Magnification Range Test}

Using a new multipurpose condenser that covers both the micro and macro range, it is possible, with the Nikon Eclipse E800, to evenly illuminate by the Köhler method all objective magnifications from $0.5 \mathrm{X}$ to $100 \mathrm{X}$. This triple condenser has three N.A. range settings that can be rotated into place either manually or automatically. Setting 1 covers objectives $0.5 X$ and $1 X_{i}$ Setting 2 covers $2 X$ and $4 X$; Setting 3 covers $10 X$ up to $100 X$.

The $0.5 X$ objective is a large, funnel-shaped lens that covers a $50 \mathrm{~mm}$ field, and provides $1 \mathrm{X}$ observation magnification. This low power high resolution objective, shown at Figure 1, eliminates not only a great deal of scanning, but also the need to switch instruments to obtain the macro range. The field it covers is more than double that of any other compound microscope lens.

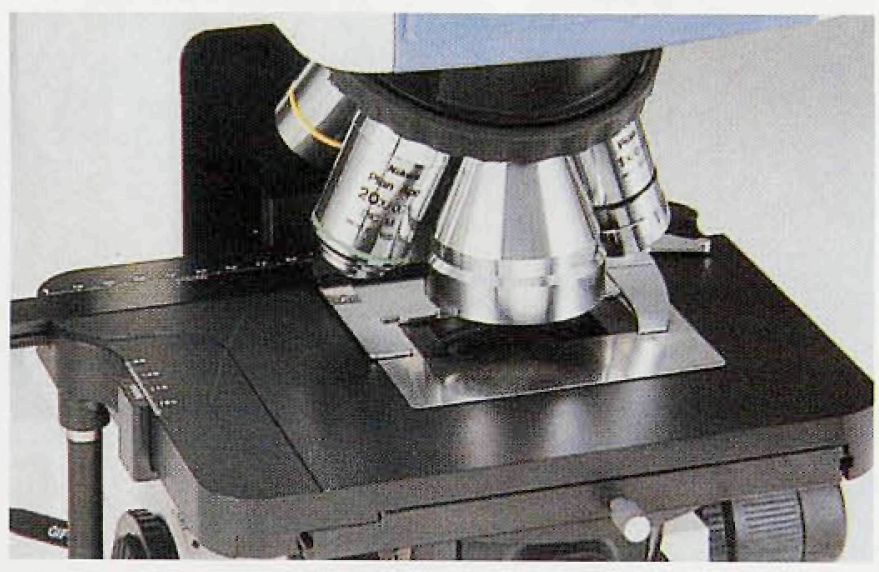

Macro Plan 0.5X Objective
Figure $2 a, b$, and $c$ show a section of a mouse head made with the $0.5 X_{1} 1 X$ and $2 X$ objectives. To further illustrate the $0.5 X$ objective, a large fluke, Prosthogonimus and a brain section of a fetal dog are shown in Figure $3 a$ and $b$.

To satisfy research protocols, it may be necessary to document the full? range of magnification available on a given specimen. Figure 4 shows a series of $\stackrel{\overrightarrow{4}}{\overrightarrow{4}}$ micrographs of a rat kidney in steps including $0.5 \mathrm{X}, 1 \mathrm{X}, 2 \mathrm{X}, 4 \mathrm{X}, 20 \mathrm{X}$ and $40 \mathrm{X}$. Blood (shown at Figure 5a) and a multipolar neuron (Figure $5 b$ ) show the $\mathrm{un}^{\mathrm{J}}$ performance of the micro-macro condenser. It takes a matter of minutes to cover the complete range of magnifications from macro to micro, all without changing a single component of the microscope.

\section{Range of Techniques}

Microscopists specified that a research instrument must provide brightfield, darkfield, phase contrast, DIC, and epi-fluorescence techniques. The epi- $\frac{\bar{\omega}}{\bar{\sigma}}$ fluorescence in the new system was like no other system evaluated. All that is $\stackrel{\circ}{\stackrel{0}{2}}$ necessary to switch from one filter cube to another is to move the lever just above $\frac{\overline{\bar{c}}}{\circ}$ the binocular housing to change excitation and emission wavelengths. All ${ }^{\square}$ fluorescence excitation wavelengths may be used simultaneously with DIC. $\frac{\hat{\rho}}{3}$ Figure 6 shows DIC performance results with Obelia Medusa(a), Diatoms(b), a 믐. Radiolarian(c), Radiolaria(d), and baby starfish(e).

Because of its modular design, the instrument can be equipped to satisfy the particular requirements of individual departments. While some work exclu- $\frac{\widehat{\Phi}}{v}$ sively in brightfield, others work primarily in epi-fluorescence and DIC, and more frequently in core facilities, work is carried out using all techniques by a variety of $\overline{D^{\circ}}$ users

\section{Ergonomics}

This brings us to the third important requirement - ergonomics. Naturally, design that prevents muscular, nerve or spinal trauma is vital. Figure 7 shows a CAD graphic of human posture/motion with the new microscope, comparing it with traditional microscopes. Note that the operator's arms here rest comfortably on the table surface, with the stage control, aperture and field diaphragms and the fine and coarse focusing knobs within fingertip reach. The binocular housing is designed for a natural viewing position.

\section{Optics}

The fourth consideration is optical and documentation facility. It is important to note that the infinity optics in the E800 and all Nikon Eclipse microscopes differ from other infinity corrected systems, in that the tube lens focal length has been increased to $200 \mathrm{~mm}$ and the parfocal distance of the objectives is increased to $60 \mathrm{~mm}$. These changes were necessary for the elimination of the optical barriers imposed by traditional systems.

Another departure from the norm is the increased diameter of the thread size of the objectives $-25 \mathrm{~mm}$, as compared to $20.23 \mathrm{~mm}$ for conventional microscope objectives. This departure from the standard has resulted in a giant step forward in the overall optical performance of the microscope. The fields viewed are so brilliant and sharp that one researcher reported that the images almost had a 3D appearance. While the micro/macro triple condenser's highest N.A. is .90 , this may easily be changed to an aplanatic achromatic oil condenser with an N.A. of 1.40 for the most critical research applications. Of course, a 1.40 condenser if used dry would have the same N.A. as the micro/macro condenser.

\section{Documentation}

One would expect that if there is anywhere that Nikon's long reputation for excellence would come into play, it would be in the area of imaging and documentation. The E800 has an unprecedented range of image capture capability, including the capacity for $35 \mathrm{~mm}$, large format $4 \times 5$, CCTV and digital recording. The photographic camera system offers many state of the art features, and is a pinnacle of design for fluorescence photography. It can be programmed to make sequential bracket exposures, double or multiple exposures, and spot and integrated readings, for instance.

Outstanding fluorescence photography is partly attributable to the system's ability to measure a spot equivalent to $1 / 1000$ of the $35 \mathrm{~mm}$ film imaging area; the instrument can also be programmed for automated brightness measurements. In both the PS and PSS modes, depending on the size of the object the researcher wishes to concentrate on, the camera automatically scans for small bright objects against the dark background and determines the optimal exposure. PS scans a 1 percent spot over an area that is 35 percent of the frame, and is preprogrammed with size and brightness referenced for appropriate exposure determination. If 
the PSS mode is chosen, a 0.1 percent spot in an area $1 / 10$ the size of that used in PS mode is scanned.

In evaluating the new instruments against the set of prerequisites that we had from lab directors and microscopists, it appears that fulfilling these users' advanced set of requirements may be a bit daunting. But the Eclipse microscope that was road tested performed beautifully against the "wish list" criteria.

As time goes on, and this new level of performance becomes the standard in research microscopy, the expectations, no doubt, will get even higher. What is next in the offing for microscopists? As it has today, our own future research needs will probably dictate the next generation of optical design and performance breakthroughs, In the interim, though, we in the field of research microscopy have a whole new world already open to us.
$2 a$

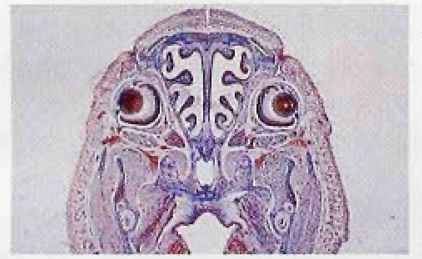

Figure 2: Section of a mouse head made with the $0.5 \times(a), 1 \times(b)$, and $2 X$ (c) objectives

Figure 3: Prosthogonimus (a) and brain section of a fetal dog (b).

3a

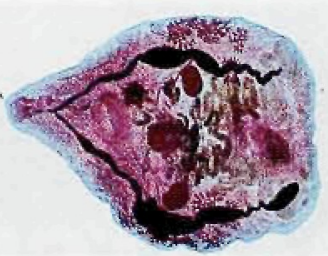

$4 a$

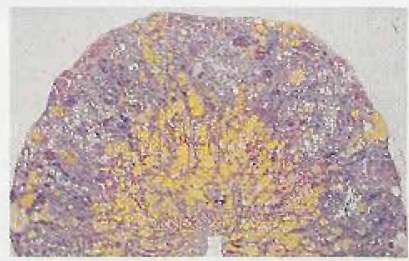

$4 c$

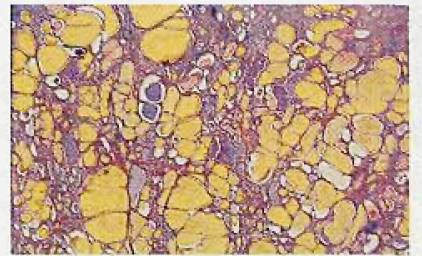

$2 b$

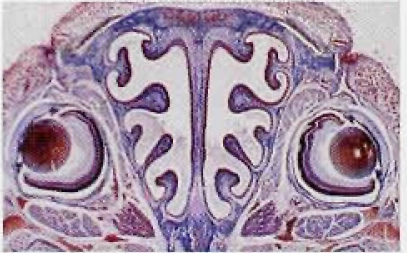

2c

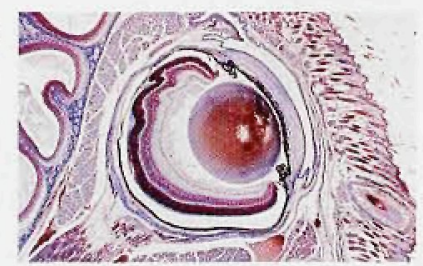

$3 \mathrm{~b}$

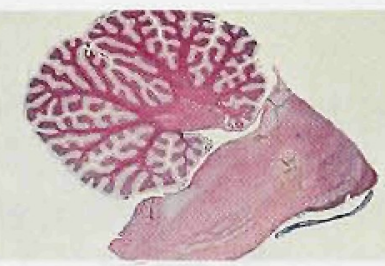

$4 b$

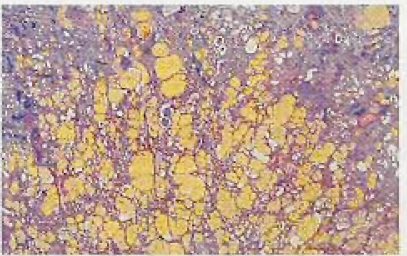

$4 d$

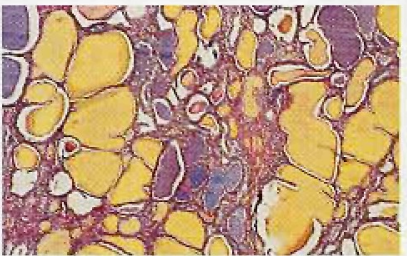

5 a

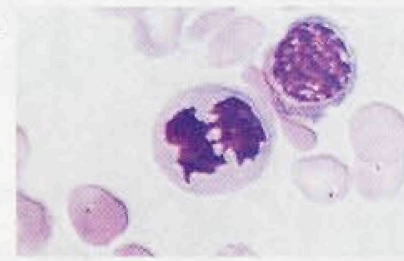

$5 b$

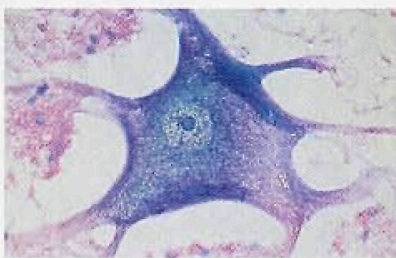

Figure 5: Blood (a) and a multipolar neuron (b) show the performance of the micro-macro condenser.
$6 a$

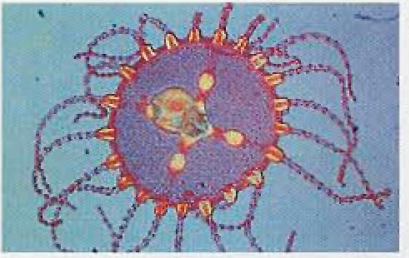

$6 \mathrm{c}$

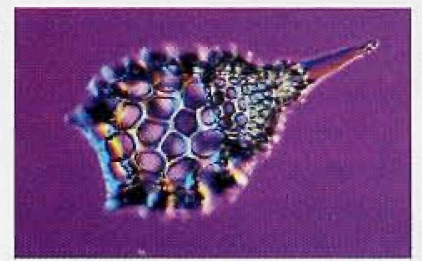

Figure 6: DIC performance results with 6 Obelia Medusa (a), Diatoms (b), a Radiolarian (c), Radiolaria (d), and a baby starfish (e). b

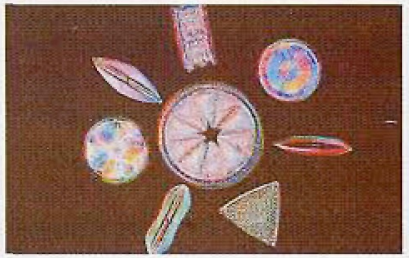

$6 \mathrm{~d}$

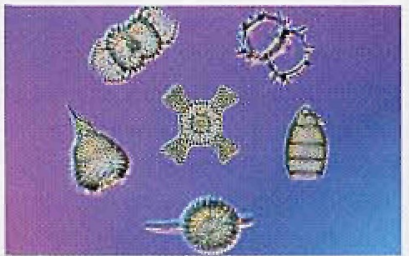

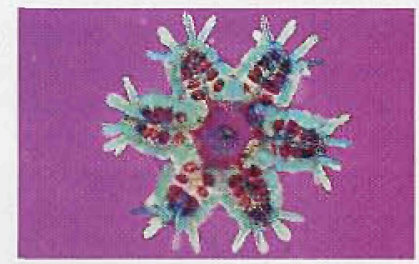

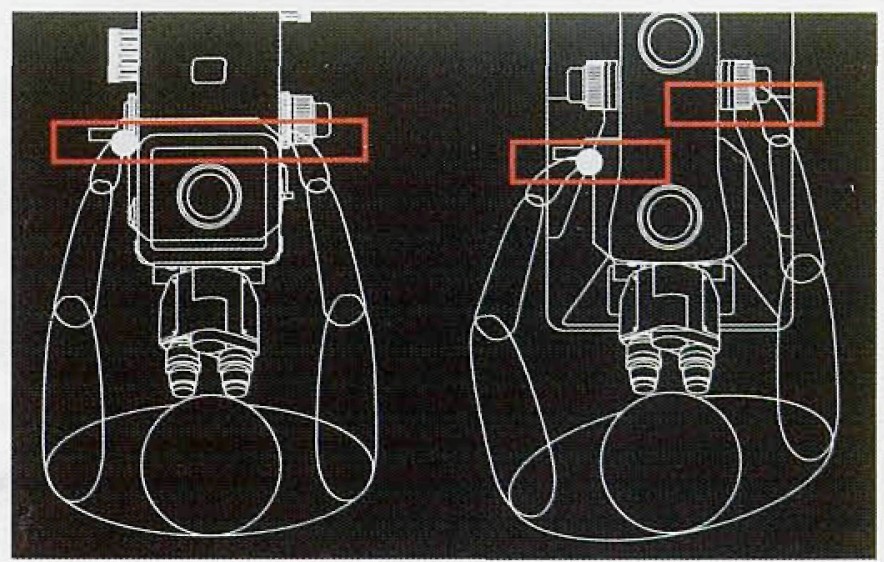

Figure 7: $C A D$ graphic of human posture/motion with the new microscope, comparing it with traditional microscopes.
$4 \mathrm{e}$

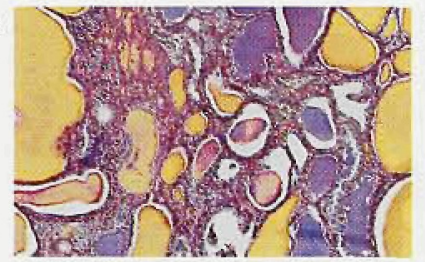

$4 f$

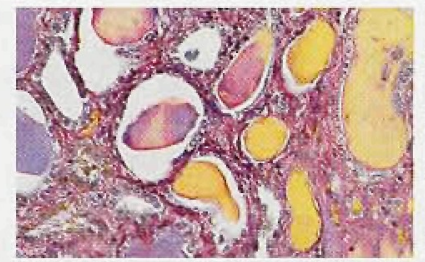

Figure 4: Micrographs of a rat kidney in steps including $0.5 X(a), 1 X(b), 2 X(c)$, $4 \times(d) 20 \times(e)$ and $40 \times(f)$.

\section{Oshel's Corollary to Heisenberg's Uncertainty Principle}

In any experiment, regardless of the results, you can never know what really happened.

This explains why a failed experiment is preferable to a successful one: It' usually possible to figure out what went wrong, but it's never possible to figure out what went right. 